\title{
Derecho PUC. Quince años de historia jurídica (1987-2003)
}

\section{Carlos Augusto Ramos Núnez}

Escribir historia solo a base de periódicos sería simplista y condenable; escribirla sin haberlos consultado resultaría, en muchos casos, temerario.

Jorge Basadre Grohmann. Introducción a las bases documentales para la Historia de la República del Perú. «Prólogo».

\section{Preámbulo ${ }^{1}$}

Numerosas revistas jurídicas aparecieron en el escenario nacional desde los inicios mismos del siglo veinte. En general, para la historia del derecho, esa producción hemerográfica — revistas profesionales y estudiantiles creadas por abogados, maestros universitarios y alumnos-, constituye un inagotable repositorio de datos que la auxilian en su tarea reconstructiva y de interpretación. Así se ha entendido entre los cultores de la disciplina, tanto en el Perú ${ }^{2}$ cuanto en el extranjero. ${ }^{3}$ En Lima, para solo señalar las publicaciones

1 Ha participado en la elaboración de este trabajo mi asistente Marcial Christian Ojeda Zaga, egresado de la Facultad de Derecho de la Pontificia Universidad Católica del Perú (PUCP). También contribuyó con su asesoría y sugerencias mi colaborador, el abogado Martín Baigorria Castillo.

2 Véanse, sobre el periodismo forense y su importancia para la historia del derecho: ClaGGeT, Helen L. A Guide to the Law and Legal Literature of Peru. Washington: The Library of Congress, 1947, pp. 14-18; BASADRE GROHMANN, Jorge. Los fundamentos de la historia del Derecho. Lima: Librería Internacional del Perú, 1956, p. 45, nota 1; RAMOS NÚNEZ, Carlos y Renzo HONORES. «Ensayos de historiografia jurídica peruana (18541937)". Cuadernos de Investigación, 1/1997. Lima: PUCP-Instituto Riva-Agüero, 1997; VARSI ROSPIGLIOSI, Enrique. "Revistas nacionales de Derecho. Consideraciones generales sobre las revistas jurídicas". El Jurista. Año 1, n. ${ }^{\circ}$, Lima, 1991, pp. 177-185; y, del mismo autor, "Las revistas de derecho en el Perú". Revista del Foro, vol. LXXX, n. 1 , Lima, 1992, pp. 325-332.

3 La utilización de las publicaciones periódicas como fuente histórico-jurídica ha sido emprendida con intensidad en otros contextos. Véase GarCía GaLlo, Alfonso. "Breve historia del Anuario". Anuario de Historia del Derecho Español. Vol. LI bis, Madrid, 
de índole legal alumbradas con posterioridad al año $1901,{ }^{4}$ destacarían $\mathrm{La}$ Revista Jurídica, vocero del Comité de Reforma Procesal (1904-1908); La Revista del Foro, órgano del Colegio de Abogados capitalino, fundada en 1914; la Revista de Ciencias Juridicas y Sociales (1927), alentada por un grupo de alumnos y profesores de la Universidad de San Marcos; la Revista de Derecho y Ciencias Politicas, órgano de la Facultad de Derecho sanmarquina, fundado en 1936 por el codificador Pedro M. Oliveira; la Revista de Jurisprudencia Peruana, creada en 1943; y la revista Derecho de la Pontificia Universidad Católica del Perú, fundada en 1944 y que es materia del presente estudio.

Renglón aparte merecen dos publicaciones que, de un $\varnothing$ u otro modo, llevaban en sí una impronta decimonónica: El Derecho, que Miguel Antonio de la Lama establece en Lima en 1885 y que se publica hasta 1909; y La Gaceta Judicial, ágil revista dirigida por Edgardo Rebagliati, de la que solo circularían seis números en 1928 y que recogía la senda de las monumentales Gacetas de 1861-1862, 1874-1876 y 1891-1893.5 Aun un periódico dedicado a ventilar los asuntos legislativos, doctrinarios y forenses existió en la capital: el Diario Judicial, que corrió bajo las órdenes de Paulino Fuentes Castro entre 1890 y 1915. En el resto del país, la proliferación de periódicos, revistas y hojas de interés jurídico fue aún más intensa. La mayor parte de estas publicaciones eran auspiciadas por los colegios de abogados de las ciudades más importantes del interior: Arequipa, Cuzco, Puno, Ayacucho, Huancayo, Huaraz, Trujillo, Chiclayo y Cajamarca. En provincias, las publicaciones jurídicas universitarias tuvieron una existencia posterior, si bien estas, al igual que en Lima, progresivamente ganarían posiciones en el avance de la hemerografía legal. $Y$ es que, si delineamos cronológicamente el devenir de estas publicaciones, hallaremos una secuencia que transcurre desde el predo-

1982, pp. VII-LIII; GrosSI, PAOLO. La Revista Juridica en la Cultura Contemporánea. Edición de Víctor Tau Anzoátegui. Buenos Aires: Ediciones Ciudad Argentina, 1997. Del mismo Grossi, "Pagina introduttiva». Quaderni Fiorentini per la storia del pensiero giuridico moderno, 16. Milán, 1987. Véase también el trabajo de Armando SOARES DE CASTRO FORMIGA. "O periodismo juridico em Portugal e no Brasil do século XIX». Versión en línea: <http://www.geocities.com/armandoformiga> (consultada el 26 de enero de 2005).

4 La producción decimonónica ha sido estudiada en detalle en RAMOS NúÑEZ, Carlos. Historia del Derecho Civil peruano. Tomo 5: "Los signos del cambio". Volumen 1: "Los repertorios legislativos y el periodismo forense». Lima: Pontificia Universidad Católica del Perú, 2005, capítulos segundo y tercero (en proceso de edición).

5 Hasta la fecha, solo se ha emprendido el indexado de la primera Gaceta Judicial, con el respectivo estudio introductorio. Véase RAMOS NÚÑEZ, Carlos. "La Gaceta Judicial (18611862)». Pensamiento Constitucional. Año IV, n. ${ }^{\circ} 4,1997$, pp. 353-418. También en separata, con idénticas señas editoriales. Se echa de menos la preparación de índices técnicamente elaborados de las Gacetas subsiguientes, así como de la revista El Derecho de 1885 a 1909. 
minio de un periodismo de carácter profesional hasta un periodismo de profesores y, por último, hasta un periodismo jurídico estudiantil.

Tal es el contexto en el que aparece la revista Derecho, órgano estrechamente ligado a la marcha de la Facultad de Derecho de la Pontificia Universidad Católica del Perú. Aquí no se quiere demostrar la importancia de esta revista. Hacia 1947, Helen L. Clagget, en un socorrido manual bibliográfico, la califica de "excelente publicación universitaria». ${ }^{6}$ Concurrían a ello dos razones: primero, que al recorrer sus páginas es posible rastrear el desarrollo del pensamiento legal peruano a lo largo del siglo $\mathrm{xx}$ y comienzos del siglo XXI. Derecho aparece, pues, como una fuente de primer orden para reconstruir aspectos esenciales de la vida social y de las ideas jurídicas desde la década de 1940 hasta la actualidad. En segundo término, discurren en la colección de Derecho PUC los temas, tendencias doctrinarias, enfoques, opciones metódicas y —last but not least-orientaciones ideológicas que, en distintos momentos, despertaron la vena intelectual de los autores.

La revista Derecho cuenta con un detallado índice general, temático y onomástico, publicado en Lima en 1990 por el Fondo Editorial de la Universidad y que incluye las primeras cuarenta entregas (abril de 1944 a diciembre de 1986). ${ }^{7}$ De manera que el presente estudio continúa esa tarea e intenta una más cuidadosa valoración de su trayectoria editorial y de la evolución de sus etapas intelectuales. Así, sin descuidar una perspectiva global, nos concentraremos en indexar y comentar el período que abarca desde el número 41 (diciembre de 1987) hasta el número 56 (diciembre de 2003) y que hasta la fecha representa la entrega más reciente de la revista.

\section{Desenvolvimiento editorial}

La Facultad de Jurisprudencia de la Pontificia Universidad Católica del Perú -antecedente de la actual Facultad de Derecho-, se funda el 29 de abril de 1919 y con ella se inicia una etapa de permanente formación de abogados y juristas en el Perú. ${ }^{8}$ En el año de 1943, en el seno de la Facultad de Jurisprudencia, nace el denominado "Seminario de Derecho", un espacio en el que los alumnos podían emprender la discusión práctica de los co-

6 Clagget, op. cit., p. 17.

7 Véase RamOS, Jorge Antonio. "Índice de la revista Derecho (1944-1986)". Lima: Pontificia Universidad Católica del Perú, 1990. La obra cuenta además de las referencias bibliográficas e índices, con un breve estudio introductorio (pp. 7-10).

- Hampe MARTínez, Teodoro. "Historia de la Facultad de Derecho de la PUCP". Manuscrito inédito. 
nocimientos teóricos que recibían. En ese contexto, se previó la formación del vocero correspondiente. Así, en el mes de octubre de 1944 aparece el número inaugural de Derecho. Organo del Seminario de la Facultad de Derecho, en un volumen de 76 páginas. Eran sus directores los profesores Hugo Piaggio Lértora (Callao 1909-1997) catedrático de Derecho Penal además de futuro decano de la facultad durante el periodo 1948-1950, y el doctor Xavier Kiefer-Marchand. Se acordó que la revista apareciera solamente durante el año académico, con una periodicidad trimestral: abril, julio y octubre.

Esta frecuencia se cumpliría sin alteración a lo largo de 1945: números 2 (abril), 3 (julio) y 4 (octubre). La saga editorial proseguiría en la entrega número 5 (abril de 1946); empero, el número 6 -aun cuando llegóa aparecer con la fecha de julio de 1946 - comprendió todo el semestre restante. No hubo un número correspondiente a octubre $y$, en adelante, las entregas virarían hacia una tendencia anual. Habría una serie de cambios en la presentación gráfica y en el aspecto general de los volúmenes. En efecto, a partir del número 33, de diciembre de 1978, y hasta el número 48 , el formato de la revista se redujo considerablemente. Evidentemente, la urgencia por reducir costos implicaba minimizar tanto el formato cuanto el número de páginas, aspecto este último que, según los mismos editores, estaría compensado por la frecuencia semestral. Secciones como «Crónica del Claustro" y el apartado de tesis se anunciaban como una publicación separada. La impresión quedaría a cargo de la universidad.

En su Indice, Jorge Antonio Ramos (encargado en varias ocasiones de la corrección de pruebas de la revista) reseña los primeros cuarenta años de la publicación y divide la historia de Derecho en tres etapas:

$\begin{array}{lll}\text { 1.a etapa 1944-1966 Números } & 1-25 \\ \text { 2.a etapa 1968-1971 Números } & 26-29 \\ \text { 3. } & \text { etapa } 1972-1986 \text { Números } & 30-40\end{array}$

El autor apela a las distintas conformaciones del mando editorial para establecer estas fases de la revista. Así, la primera etapa se caracterizaría por contar con directores. Es la etapa de Piaggio Lértora y Kiefer-Marchand, que asombra por su duración (22 años) y durante la cual se lograron editar las primeras 25 ediciones. En la segunda etapa, que transita entre los años de 1968 y 1971, desaparece el cargo de director, que es reemplazado por un editor responsable y un consejo editorial. Luis H. Pásara Pazos (Lima, 1944) fue el primer «editor"; y sus asistentes, la señorita Martha Chavarri Dupuy y el señor César Arias Quincot. El primer consejo editorial lo integrarán, a partir del número 29 (octubre de 1971), Domingo García Belaunde (Lima, 1944), Alfredo Ostoja López Alfaro (Lima 1941-1990) y el citado Luis 
Pásara. A partir de la tercera etapa, que, según Ramos, comprendería los años de 1972 y 1986, las responsabilidades editoriales descansan principalmente en los docentes de la Facultad de Derecho. De manera que, si en sus comienzos Derecho nace como un canal de expresión de la producción estudiantil, ahora tenía como su principal objetivo la difusión del pensamiento docente, aunque sin descuidar, naturalmente, la presencia de los mejores trabajos de los estudiantes.

Cabe, sin embargo, una precisión: si hubiéramos asumido el criterio del régimen editorial adoptado por Jorge Antonio Ramos, la «tercera etapa" de Derecho debería extenderse también a los números 41 a 48 (diciembre de 1987 a diciembre de 1994), los que, como es natural, no figuraron en el espectro temporal del Índice publicado en 1990. De modo que tendríamos:

1. a etapa 1944-1966 Números 1-25

2. a etapa 1968-1971 Números 26-29

3. a etapa 1972-1994 Números 30-48

En 1995, las autoridades de la Facultad de Derecho confían el cargo de editor general de la revista a Aníbal Quiroga León, quien estaría al frente de la publicación hasta el año 2003. Merced a los cambios _principalmente formales - introducidos por la nueva administración, podría afirmarse que, a partir de aquel año, la revista ingresa en una cuarta etapa. Precisamente, en la presentación del número 49 , el flamante editor general anuncia el inicio de una nueva época en la trayectoria de la publicación. Quiroga ensaya incluso una periodificación alternativa de la revista: la primera etapa de Derecho correría de 1944 a 1966 , es decir, el período coincidente con la dirección de los doctores Piaggio y Kiefer-Marchand; la segunda etapa la sitúa Quiroga en el lapso 1968-1974, que estaría caracterizado básicamente por el cambio en el formato de los volúmenes; la tercera época, que comprendería el período 1978-1994, se corresponde con un tamaño aún más reducido:

$$
\begin{aligned}
& \text { 1. a etapa 1944-1966 Números 1-25 } \\
& \text { 2. a etapa 1968-1974 Números 26-32 } \\
& \text { 3. a etapa 1978-1994 Números 33-48 } \\
& \text { 4. a etapa 1995-2003 Números 49-56 }
\end{aligned}
$$

Esta periodificación, como veremos luego, resalta también el silencio editorial de casi cinco años que separa los números 32 (diciembre de 1974) y 33 (diciembre de 1978). 
Quiroga asumió sus labores de manera simultánea con el cincuentenario de la revista, efemérides que se presentaba como el marco perfecto para iniciar un proceso de reformas. Se abandonó, por razones de presupuesto, el formato estándar de $15,5 \times 20 \mathrm{~cm}$, que se observa desde el número 33 y que se conservaría hasta el número 48, de diciembre de 1994. A partir de la entrega número 49 se optó por el tamaño de $24,5 \times 16,5 \mathrm{~cm}$. Los volúmenes se imprimían en papel bond, con una portada polícroma plastificada. El emblema de la universidad ornaba cada una de las ediciones. No obstante dichos cambios, se mantuvo la tradición, instaurada desde el número 37 (diciembre de 1983), de ofrecer en la cubierta (e incluso en la contracarátula) el sumario con los nombres de los colaboradores y sus respectivos aportes, tradición que se conserva invariable hasta hoy.

Un cambio de mayor monta, y sin duda el más evidente, alude al nombre de la revista. Al parecer, el nuevo consejo editorial se había percatado de que la denominación Derecho era idéntica a infinidad de publicaciones afines, tanto nacionales cuanto extranjeras. Esto no hacía más que agravar la confusión bibliográfica y, naturalmente, disminuía el espíritu distintivo que la revista intentaba traducir. No hubo mejor idea que recalcar este propósito con una adición al título originario. Surge así la nomenclatura compuesta: Derecho PUC, con la cual se le conoce en la actualidad. Los frutos de la etapa 1995-2003 fueron ocho ediciones, además de dos números extraordinarios. El primero de ellos estuvo consagrado al octogésimo aniversario de la Facultad de Derecho; el segundo número extraordinario fue lanzado en coedición entre el Fondo Editorial de la PUCP y el American Law Institute, entidad que guarda consistente relación con la universidad. ${ }^{9}$

Una de las aspiraciones que definen el período 1995-2003 fue lograr la exacta frecuencia semestral de la revista. Pero existía un escollo: el equilibrio cronológico se veía afectado debido a algunas discontinuidades en la periodicidad. ${ }^{10}$ Ambición de por sí muy ardua y a la.larga no materializada, este

9 Véase Derecho PUC, n. ${ }^{\circ}$ 2, diciembre 1998-marzo 1999; edición especial: "Proyecto de Normas Transnacionales del Proceso Civil. Transnational Rules of Civil Procedure». Esta edición contiene solo tres artículos provenientes del número 52 . El tema principal es la uniformización de las reglas generales del common law y del civil law. Los conceptos de "proceso» y "globalización» son analizados por los profesores Geoffrey Hazard (Universidades de Pensilvania, Yale y Berkeley) y Michele Taruffo (Universidad de Pavia), quienes presentan su "Proyecto de normas transnacionales del proceso civil», con presentación del profesor Antonio Gidi (Universidades de Bahía y Pontificia Universidad Católica de São Paulo, Brasil). Geoffrey Hazard también figura con una contribución sobre "Litigio civil sin fronteras: Armonización y unificación del Derecho Procesal».

10 La discontinuidad mayor que sufriera la revista se extendió a lo largo de los años 1975, 1976 y 1977, en los cuales no apareció ningún número. Otro lapso, como anticipamos, ocurre luego de la salida del número 32 de noviembre de 1974 y el número 33 de diciembre de 1978, cuatro años sin ediciones, hecho que, en el futuro, incidiría en una 
hecho no resta mérito a ninguna de las ediciones que se consiguió entregar. La revista amenguó su periodicidad pero, a la par, compensó esta situación con el aumento progresivo en el número de páginas. Hasta cierto punto puede decirse que, en esta etapa, la historia de la revista es también la génesis de su crecimiento físico o, mejor dicho, de sus dimensiones. Si comparamos el esquema organizador de Aníbal Quiroga con la tercera época, caracterizada por la brevedad, comprobaremos que esta fase está signada por el gigantismo. El Consejo Editorial de Derecho PUC empezó sus funciones con la participación de Domingo García Belaunde, Baldo Kresalja Roselló y Enrique Lastres Berninzon para sus tres primeras ediciones: número 49 (diciembre 1995); número 50 (diciembre 1996); y número 51 (diciembre 1997). Kresalja y Lastres habían conformado ya el consejo directivo precedente, ${ }^{11}$ mientras que la edición número 49 contó - por única vez - con la participación del profesor Lorenzo Zolezzi Ibárcena, a la sazón decano de la Facultad, lo que sin duda expresaba un apoyo institucional al relanzamiento editorial de la revista.

Con la aparición del número 52 (diciembre 1998 - abril 1999), el Consejo Editorial se renovaría. Ingresan los profesores César Guzmán Barrón, José Carlos Ugaz Sánchez-Moreno y Francisco Avendaño Arana, conformación que se mantendría inalterada hasta la penúltima edición, número 55 , de diciembre de 2002. Anotemos que en la edición número 56, no se hace constar la conformación del Consejo.

\section{Tendencias, métodos, enfoques}

Del examen de los índices de Derecho y Derecho PUC se advierten tres fases en su existencia editorial. A la primera etapa, que va desde su fundación en 1944 hasta el año de 1966, la podríamos calificar de "cultural" y conservadora. Predomina la relación entre el derecho y las concepciones humanísticas, así como una clara adhesión a la doctrina de la Iglesia. El pensamiento católico asoma en el tratamiento de temas como el divorcio y sus causales. La presencia en los claustros de Víctor Andrés Belaunde - fundador del Mercurio Peruano- resulta gravitante. La segunda etapa de Derecho es la del cambio social, en la que se pregonan las tendencias desarrollistas, la teoría de la dependencia y la doctrina social de la Iglesia, que son defendidas por autores como Luis Pásara, Fernando de Trazegnies, Jorge Avendaño, Marcial Rubio Correa, Lorenzo Zolezzi, Domingo García Belaunde, Alberto Bustamante,

irregularidad tal que, por lo general, producía una discrepancia de un año entre la fecha de edición y el año efectivo de salida de imprenta.

"Véase Derecho PUC, n. ${ }^{\circ} 46$, diciembre de 1992. 
Guillermo Figallo y el propio Héctor Cornejo Chávez, entre otros. Esta etapa, que corre desde 1967 hasta 1980 aproximadamente, sería coincidente con el gobierno militar de Velasco y Morales Bermúdez. Desfilan, por ende, temas tales como la reforma agraria, la comunidad industrial, el tránsito hacia el socialismo, la enseñanza del Derecho, la democracia representativa o el papel del derecho en la sociedad. La tercera etapa, definida por una suerte neutralidad técnica, podría fijarse desde 1980 hasta la actualidad. Esta exhibe la notoria preocupación de los colaboradores por señalar los defectos de la legislación y la consiguiente urgencia por esbozar los nuevos códigos hoy existentes (a saber, el Código Civil de 1984, el Código Penal de 1991, el Código Procesal Civil de 1993, la Ley General de Sociedades de 1999, el Código Procesal Constitucional de 2004 y el Código Procesal Penal de 2004), en cuya génesis los profesores de la Facultad de Derecho de esta casa superior de estudios tuvieron una responsabilidad fundamental.

Otros campos de reflexión que hallan cabida en la fase actual de Derecho PUC son la divulgación del pensamiento constitucional; la independización de los derechos humanos frente al derecho civil y constitucional, y su emergencia como objeto de una disciplina autónoma; el surgimiento de los llamados "derechos del niño y del adolescente" (repentinamente, digamos de paso, se abandona la categoría jurídica de menor por la convencional de niño, en cuanto se asume que la primera denota una cierta restricción de capacidad jurídica); el entusiasmo por el tema de los derechos del consumidor y la libre competencia; la simplificación del derecho tributario y administrativo; el medio ambiente. Un rasgo curioso: asoma la presencia del Law and Economics, pero con mucha menor incidencia que en las revistas de los estudiantes, Ius et Veritas y Thémis. Cabe decir que todas las mencionadas constituyen corrientes y hasta modas intelectuales que los juristas del derecho positivo en cada generación abrazan con entusiasmo, sin advertir las tendencias subyacentes. $Y$ es que una moda - por definición- está destinada a aparecer, extenderse y decaer inexorablemente, para ser reemplazada por otra que, a la larga, correrá la misma suerte.

\section{Derecho PUC, número a número}

\section{Número 41}

Tenemos que, para el año de la salida de la edición 41, correspondiente a diciembre de 1987, la impresión se finalizó en agosto de 1988. El Consejo Editorial para ese entonces estaba conformado por Miguel de Althaus y Marcial Rubio (Lima, 1948) como profesores principales, además de Iván 
Jara Flórez en calidad de profesor auxiliar. Se contaba, además, con la presencia de un representante estudiantil que en esa oportunidad fue el señor Fernando García Granada. La designación de este último la realizaba el tercio estudiantil de la Facultad de Derecho.

Debido a la creación de un magíster de Derecho con mención en Derecho Internacional Económico —el primero que se impartió-, la sección central no encontró mejor manera de presentarse que incluyendo trabajos realizados por autores foráneos. De esta manera, tenemos la publicación de trabajos como el de la profesora de Derecho Internacional en la Universidad de Chile, María Infante, titulado "La solución judicial de controversias entre Estados». Esta colaboración estuvo estrechamente ligada a su presencia como profesora visitante en la maestría referida, en la cual tocó el tema de la solución de controversias.

Al mismo tiempo, esta edición pudo contar con el trabajo del profesor de la Universidad de Buckingham del Reino Unido, Phillips James. Él, con sus "Cuatro conferencias sobre la Ley de Torts" -obra resultado de dos ciclos de charlas dictadas el año de 1986-, nos ubica, con una introducción panorámica, en el razonamiento y lógica con la que se conduce el derecho inglés en general. Posteriormente, pasa a dar una explicación de cómo esta ley se relaciona con la teoría del daño y su relación con acciones como la negligencia y la difamación.

Cabe resaltar la publicación del voto disconforme que hiciera en ese entonces el ministro de la Corte Suprema Argentina, Jorge Bacqué, ${ }^{12}$ respecto de la Ley de Obediencia Debida, causa entablada en directa relación con el decreto 280/ 84 del Poder Ejecutivo Nacional Argentino. Este declara expresamente la inconstitucionalidad de una ley configurada manifiestamente con fin de exculpar de responsabilidad a los miembros de las Fuerzas Armadas Argentinas que actuaron con una estrategia de "guerra sucia» para represar el terrorismo, que violaron múltiples derechos fundamentales y que trataron de justificar estos atropellos como parte de la defensa de los intereses nacionales y la integridad territorial.

Además de los valiosos aportes señalados, no faltaron, en esta edición, participaciones de representantes nacionales en el área internacional, como "La mediación: teoría y práctica» obra presentada por Eduardo Ferrero, ${ }^{13}$ como también la contribución de juristas de otras áreas como es el caso de

12 El doctor Bacqué es un jurista argentino que se desempeñó como ministro de la Corte Suprema Argentina desde el año de 1985. Fue apartado de la docencia que ejercía en la Facultad de Derecho de la Universidad de Buenos Aires en 1976, época de severa represión a la libertad de cátedra.

13 Catedrático en el área de Derecho Internacional en la PUCP y Presidente Ejecutivo del Centro Peruano de Estudios Internacionales (CEPEI). 
entonces joven estudiante del Magíster en Derecho, Gastón Fernández Cruz, "La buena fe en la concurrencia sobre bienes inmuebles». ${ }^{14}$

Para ese tiempo, la sección "Crónicas del Claustro" nos daba cuenta de la elección como decano, en junio de 1987, del doctor Jorge Avendaño, auxiliado por el Consejo de Facultad compuesto por Guillermo Velaochaga, Felipe Osterling (Lima, 1932), Roger Rodríguez Iturri y Marcial Rubio.

También se da registro de la conformación de la población estudiantil del momento al describirse: «Actualmente la Facultad de Derecho de la Pontificia Universidad Católica tiene matriculados 1,600 alumnos y presta servicios de enseñaza a otros 400 procedentes de otras unidades académicas de la Universidad.$^{15}$ Teniendo en cuenta estas cifras —elevadas para la época- se manifiesta: "[...] ante tal situación, una de las tareas más urgentes es la de lograr que la Facultad cuente con un local que tenga capacidad suficiente para albergar a sus profesores, alumnos y personal administrativon. ${ }^{16}$

\section{Número 42}

Derecho 42, correspondiente a diciembre de 1988 , contó para su elaboración con el mismo consejo editorial de la edición anterior. ${ }^{17}$ Se tuvo en esta oportunidad como sección central la presentación de artículos de los alumnos de otro Magíster realizado, pero en esta ocasión con mención en Derecho Civil entre los años de 1987 y 1988. Esta edición pretendía divulgar los trabajos más destacados de los alumnos, según el criterio de los profesores encargados de la selección. Este proceso dio como resultado contar con artículos como «Algunas reflexiones acerca de la inseminación artificial y la fecundación extrauterina» presentado por Carlos Cárdenas Quiros. Él, planteando los problemas jurídicos que se originan con la utilización de la inseminación artificial y la fecundación extrauterina, analiza las legislaciones extranjeras - con énfasis en la legislación europea- a fin de que a partir de este trabajo se produzcan posteriores enfoques al respecto. También se tiene registro del aporte

14 Catedrático en Derecho de Obligaciones en las Universidades de Lima y San Marcos Egresado de la Facultad de Derecho de la PUCP y participante en ese momento del Magíster en Derecho Internacional Económico.

15 "Crónica del Claustro». Derecho, n. ${ }^{\circ} 41$. Revista de la Facultad de Derecho de la PUCP, Lima, 1987, p. 477.

16 Ibídem, p. 478. Al respecto se inicia una campaña que involucraba llamar a la colaboración entre docentes y egresados con la finalidad de captar los fondos necesarios que puedan ser destinados a concretar el anhelo del proyecto del nuevo local de la Facultad de Derecho.

17 La única variante esta vez fue la presencia del representante estudiantil Christian Schroder Romero. 
de Roger Rodríguez Iturri, ${ }^{18}$ quien con su trabajo "Matrimonio y familia: información sobre el parentescon conjuga la investigación jurídica con referencias de corte histórico y antropológico para poder así configurar el criterio de afinidad que delimita el ordenamiento civil peruano.

En una sección denominada como "Varia" destacó el trabajo de Domingo García Belaunde, denominado «Sistema Electoral y representación política en el Perú actual", que había sido producto de una publicación parcial en Derecho 41 y que, en esta oportunidad, se reprodujo en su integridad. Este trabajo analiza el proceso de maduración del sistema legal electoral peruano, apelando a estadísticas y porcentajes de representatividad con los cuales el autor deduce que la legalidad establecida desde 1963 con procesos electorales indiscutidos se mantuvieron incluso durante el periodo del gobierno militar (1968-1980). Este, al generar conciencia política en sectores marginados, logró el efecto de la conciencia electoral que significó una asistencia electoral masiva a los diversos procesos sucesivos.

Al mismo tiempo, las crónicas de la Facultad daban cuenta destacada de un nuevo reglamento en ella, uno que traería novedades como la introducción de prácticas profesionales como parte y requisito de la formación universitaria, y la precedencia de los jurados en los exámenes para la obtención de los grados de bachiller y título universitario. Al mismo tiempo, el doctor Héctor Cornejo Chávez (Arequipa, 1918), luego de treinta años de enseñaza, recibía la distinción como Profesor Emérito. Asimismo, lo que nació como una iniciativa para construir el ansiado pabellón de Derecho se convirtió en el proyecto Proderecho, un movimiento encargado de recibir los aportes que ya consolidaban sus primeros frutos con la construcción de las primeras ocho aulas. ${ }^{19}$

\section{Números 43-44}

Derecho 42 terminó de imprimirse en enero de 1990. Este hecho, sin duda, generó una discontinuidad que corría el riesgo de acrecentarse. Ante esto se procedió a editar una edición que intente evitar esta amenaza. Con la salida de Derecho 43-44, perteneciente a diciembre de 1989 y diciembre de 1990, se colaboró con tal fin, teniendo en cuenta que la salida de imprenta de esta entrega ocurrió en abril de 1991.

\footnotetext{
18 Profesor de la Cátedra de Derecho Civil quien, para el tiempo de edición, ejercía el cargo de Director de Estudios de la Facultad de Derecho.

19 "Crónica de la Facultad de Derecho». Derecho. Revista de la Facultad de Derecho de la PUCP, n. ${ }^{\circ} 42$, Lima, 1988, p. 321.
} 
Esta edición, a diferencia de las demás, contiene un editorial más extenso en tanto contiene una breve reseña de los artículos que se estaban incluyendo; de esta manera, supera el breve apartado que, bajo el titulo «De los editores", se remitía a información básica del contenido en las ediciones anteriores. Este primer editorial estuvo firmado por el doctor César Landa con quien, a su vez, se retomó la aparición del cargo de director.

Este número estuvo dedicado a la memoria del profesor Alfredo Ostoja López Alfaro, fallecido el 30 de abril de $1990 .{ }^{20} \mathrm{La}$ edición tuvo como tema principal el homenaje por el décimo aniversario de la puesta en vigencia de la Constitución peruana de 1979. Entre los artículos que conformaron este número se puede apreciar la diversidad de especialistas en materia constitucional con las que cuenta la Facultad de Derecho por la variedad de aportes que se presentan: textos como el de Domingo García Belaunde «El constitucionalismo peruano en la presente centurian, ${ }_{2}^{21}$ que hace una revisión histórica del desarrollo constitucional peruano. Para ello se vale de un estudio preliminar al cual le sucede una presentación de los periodos históricos de la historia constitucional peruana, con un análisis de una época llamada "Constitucionalismo Social" que abarcó las cartas de 1920, 1933, que finaliza con la de 1979 y que, en palabras del autor, simboliza la «continuación y negación, al mismo tiempo, del gobierno militar». Destacan también el respaldo a los derechos humanos, la ratificación y ampliación de los mecanismos procesales constitucionales actuales, los intentos en la lucha por la consagración de un modelo regional y la superación del enfoque semiparlamentarista que presentaba la carta de 1933 por un reforzamiento de la investidura del jefe de Estado, hecho que fomentaba la tendencia al presidencialismo.

Apreciable también resultó el aporte de Francisco Eguiguren Praeli (Lima, 1953) quien, analizando la década producto de homenaje (1980-1990) en su texto «Desarrollo y vigencia de los derechos y garantías en diez años de régimen Constitucional», asevera en una frase que el Perú «tuvo muchas Constituciones y poca constitucionalidadm. ${ }^{22}$

2o Alfredo Ostoja (Lima 1941-1990) fue docente en la Facultad de Derecho, además de pertenecer a la historia de la revista Derecho al haber conformado parte del Consejo Editorial durante los años 1971 y 1981.

21 Texto concluido en junio de 1987, preparado para la edición conmemorativa por los setenta años de la vigencia de la Constitución mexicana en 1917. Homenaje Universitario de la UNAM. El trabajo presentado para Derecho es una versión adicionada y corregida.

2 Ya adentrándose en el modelo político que planteaba la Carta de 1979, marcado por el ideario del "Estado social y democrático de derecho" que, sin embargo, para la época en niveles de verdadera aplicación y concreción aún quedaban en deuda por su falta de efectividad y viabilidad política. Aspectos como la ineficacia de un modelo de respeto a la libertad personal, por actitudes arbitrarias de las autoridades policiales y políticas en 
Por otro lado, las crónicas de Derecho nos anunciaban, junto con los apartados administrativos, que, en casi dos años de captación de recursos financieros, el proyecto de la construcción de la nueva Facultad de Derecho ya se concretizaba con un nuevo pabellón de ocho aulas. Estas tenían capacidad para 110 alumnos cada una, lo que sin duda marca un paso importante en la historia de la facultad. ${ }^{23}$

\section{Número 45}

Quizá un momento decisivo en la dinámica editorial de la revista haya sido el año de 1991. En efecto, la entrega de Derecho 45 debería pertenecer a diciembre de ese año. En un gran esfuerzo por lograr la equiparidad cronológica de entregas anuales, se logró que esta edición saliera de imprenta en octubre del mismo año. Sin duda, este fue un gran esfuerzo del Consejo Editorial, que contaba en esta ocasión con la dirección de César Landa, asistido por los profesores Jorge Avendaño, Francisco Eguiguren y la representante estudiantil Giovanna Yataco. Se vivían años de transición política que, sin embargo, no hacían presagiar los cambios radicales que sucederían en el orden democrático. De esta manera, la inquietud académica y el análisis de Derecho, al dedicar recientemente un apartado reservado al área constitucional con su edición anterior, quería redireccionar su estudio abordando el Código Civil. Una publicación referencial en este sentido es Derecho 37, que se realizó un año antes de la promulgación de Código Civil de 1984 y que presentó artículos que analizaban este ordenamiento aún en su fase de proyecto. ${ }^{24}$

Se reiteraban, en aquel número, las condolencias por la desaparición del doctor Ostoja pero también se dedicaba la edición a la sentida c'esaparición, en el mes de julio de 1991, de Francisco Interdonato, sacerdote jesuita que

el Perú de los ochenta, los prolongados estados de emergencia, las ejecuciones extrajudiciales por las fuerzas de seguridad y las acciones subversivas latentes aún en la década de los noventa. Así como la politización en el uso de la demanda de amparo y el criticable aspecto de suspensión provisional del acto reclamado que atentaban contra su efectividad. Si a esto se unía la ineficacia de la institución del hábeas corpus en un país de precaria eficiencia técnica de un poder judicial para su efectivización.

Además de una crítica a la discreta actuación del entonces Tribunal de Garantías Constitucionales, aspecto reflejado en el debilitado y casi imperceptible uso de los sistemas de Acción de Inconstitucionalidad y la Acción Popular.

23 Derecho, n. ${ }^{\text {os }} 43-44$, p. 463.

${ }^{24}$ Se pueden encontrar trabajos como los de RODRíGuEZ ITURRI, Roger. "Algunas anotaciones al libro de familia del Proyecto de Código Civil", pp. 187-213; CÁrDENAS QuIROS, Carlos. "El derecho real de la superficie», pp. 7-30 y la obra de MARCENARO FRERS, Ricardo. "La igualdad de derechos del hombre y la mujer en el Código Civil vigente y el proyecto», pp. 95-101. Derecho, n. ${ }^{\circ}$ 37, Lima, diciembre 1983. 
participó de la historia de Derecho al haber colaborado con dos publicaciones, ambas en la década de los ochenta ("Relaciones de la Iglesia y el Estado en la nueva Constitución del Perú»" ${ }^{25}$ "Concepto y realidad de la Ley Natural»). ${ }^{26}$ Ya que no se trataba de un jurista (fue doctor en Filosofía y Teología, además de catedrático en esta última rama desde 1970 en la Pontificia Universidad Católica), estas obras manifiestan la admisión que daba Derecho a la investigación, incluso con otras ópticas, de los diversos fenómenos jurídicos.

En lo que se refiere al contenido de esta edición, se tienen entregas como un adelanto de lo que se convertiría en la tesis de magíster del profesor Gastón Fernández Cruz, con un análisis y discusión sobre la naturaleza jurídica del interés y de los defectos que poseen las clases de interés presentadas en el Código Civil de 1984. ${ }^{27}$ También se puede ver la recuperación de la doctrina de los actos propios como criterio de aplicación del principio general de la buena fe en el ordenamiento civil, obra que realiza René Ortiz Caballero. ${ }^{28}$ También se exhibe un trabajo de Miguel Torres Méndez, quien hace un cuestionamiento sobre cuál sería el posible régimen de transferencia de propiedad de los bienes muebles incorporales en nuestro Código Civil. ${ }^{29}$

Un tema controversial, que remite al ámbito jurídico y conexamente al plano moral, es el que plantea Beatriz Boza al analizar la reproducción humana asistida y su relación con el derecho. La posición de la autora es categóricamente conservadora cuando sostiene que esta tecnología debe ser privilegio restrictivo: «Solo las personas casadas fértiles e infértiles podrán beneficiarse de los adelantos de la ciencia». Se excluían de esta manera a los convivientes o a las personas libres. Sustenta su postura en la defensa de la

25 INTERdonato, S. J., Francisco. "Relaciones de la Iglesia y el Estado en la nueva Constitución del Perú". Derecho, n. ${ }^{\circ} 35$. Lima, junio 1981, p 87-95.

z INTERdonato. S. J., Francisco. "Concepto y realidad de la Ley Natural». Derecho, n. 41. Lima, diciembre 1987, pp. 267-290.

27 FERNÁNDEZ CRUz, Gastón. "La naturaleza jurídica de los intereses: punto de conexión entre Derecho y Economía». Derecho, n. ${ }^{\circ} 45$. Lima, diciembre 1991, pp. 177-213.

23 Ortiz Caballero, René. "La doctrina de los actos propios en el derecho civil peruano". Derecho, n. ${ }^{4} 45$. Lima, diciembre 1991, pp. 265-285.

29 El planteamiento inicial del autor señala que el Código Civil de 1984 no regula el modo de transferencia de propiedad aplicable a los bienes muebles incorporales. El autor explica que el modo de transferencia de la propiedad se concretiza propiamente por la "cuasitradición" entendida como entregar el bien incorporal por simple otorgamiento de escritura pública para los muebles incorporales, aspecto este que no es regulado expresamente. La analogía, por tanto, hace que se use la tradición documental para salvaguardar el vacío legal. Véase TORRES MÉNDEZ. Miguel. "La transferencia de propiedad de los bienes muebles incorporales en el Código Civil». Derecho, n. ${ }^{\circ}$ 4. Lima, diciembre 1991, pp. 159-175. 
familia como célula básica de la sociedad.$^{30}$ Hoy, esta sería una tesis indefendible.

\section{Número 46}

Con una renovación del Consejo Editorial que, además del profesor César Landa —ahora flamante magistrado del Tribunal Constitucional-, contaba en esta ocasión con los profesores Enrique Lastres, Baldo Kresalja y el representante estudiantil Henry Huerta, se pudo alcanzar a la comunidad jurídica Derecho 46, correspondiente a diciembre de 1992 con una salida de imprenta que correspondió a marzo de 1993. Para entonces, la situación política del país pasaba sus momentos más delicados después del autogolpe del cinco de abril y la consiguiente derivación de sucesos atentatorios contra el Estado de derecho y la garantía de independencia de las instituciones estatales. Por cuestiones de edición, Derecho 46 no pudo prever estos acontecimientos en concretos artículos que analizaran el nuevo panorama nacional, dado que el trabajo recopilatorio de artículos, así como el compromiso de edición para con sus autores habían asumido el adeudo de hacer de Derecho 46 una edición dedicada fundamentalmente al Derecho Penal. Ello, teniendo en cuenta la importancia de la puesta en vigencia del Código Penal de 1991, aspecto que, sin duda, generaría diversos cambios en el orden penal peruano que merecían ser tratados. Sin embargo, esto no fue óbice para que, en un apartado denominado «Nota del Director", César Landa se pronunciara. A propósito cita a Manuel Atanasio Fuentes, quien hacia 1873 manifestó que ni los diversos problemas institucionales ni ninguna otra justificación validaban jurídicamente el rompimiento del orden constitucional establecido. ${ }^{31}$

3 La autora descarta a los solteros y a los homosexuales y, en virtud a lo establecido por el artículo 289 del Código Civil, que sanciona el débito sexual conyugal y la fidelidad recíproca que también refiere el artículo 288, se llegaría a la conclusión que en el marco de una unión estable y legitimada deben limitarse las técnicas. Véase BOzA DiBOS, Beatriz. "Los adelantos de la ciencia y la permeabilidad del derecho: reflexiones en torno a la Reproducción Humana Asistida" Derecho, n. ${ }^{\circ} 45$. Lima, diciembre 1991, pp. $71-110$.

31 "Nota del Editor". Derecho, n. ${ }^{\circ}$ 46. Lima, diciembre 1992, pp. 6-9. La frase textual fue «El Perú es un conjunto de pueblos, siempre oprimidos, siempre confiados en nuevas y pomposas promesas y siempre burlados". Introducción perfecta para que César Landa afirme que la desactivación del Congreso e instituciones afines al control político por un poder ejecutivo apoyado por sectores empresariales, públicos, de comunicación y las Fuerzas Armadas, no podían basar el quebrantamiento del Estado de derecho. Se culmina clamando por la exigencia de la reactivación de la institucionalidad autónoma de los poderes legislativo y judicial si realmente la labor del Congreso Constituyente Democrático que emergía buscaba tener legitimidad democrática. 
Presentadas las justificaciones correspondientes, trascienden en esta edición penalista contribuciones como las de Luis A. Bramont Arias, con un análisis de los principios que regirían el Código Penal de 1991 que entraba en sustitución de su par de 1924;32 estudios como los de César San Martín Castro, en el que se analiza el desenvolvimiento judicial y policial en los casos de narcotráfico; ${ }^{33}$ o el de Víctor Prado Saldarriaga que cataloga "la pena" como mecanismo de intimidación social y castigo; y la califica de autoritaria, muy alejada de la intención constitucional de instrumento resocializador y preventivo. Este artículo destaca porque de él se desprende que el Perú tiene "la pena" como muestra del poder de represión que ejerce el Estado, al anteponer así, ante la sociedad, su necesidad de estabilidad política por sobre lo establecido en la Constitución. ${ }^{34}$ En la misma dirección de análisis estuvo la contribución de Felipe Villavicencio Terreros con su trabajo sobre el tratamiento del Código Penal al hecho punible. ${ }^{35}$

\section{Número 47}

Derecho 47 contó para su elaboración con la conformación íntegra del Consejo Directivo de la edición anterior y correspondía en esta ocasión a diciembre de 1993. Su impresión fue realizada en marzo de 1994. Las Crónicas del Claustro nos dan cuenta de un acuerdo de facultad con fecha 31 de marzo de 1993 en la cual se establece la instauración de una placa conmemorativa en distinción y recuerdo del Decanato que ostentara entre 1964 1970 y 1987-1993 Jorge Avendaño Valdez. Además, se informa de la institución, a partir del primer semestre universitario de ese mismo año, del sistema de Secigra como parte de la formación de los estudiantes de la

32 Un estudio del Título Preliminar hace inferir que el autor que este nuevo Código Penal da visos de no haberse apegado totalmente a una escuela penal en particular. Se abría una época de decretos leyes para casos de terrorismo, juzgamiento en ausencia, jueces sin rostro, suspensiones de hábeas corpus, jurisdicción militar, aumento de atribuciones decisorias del Ministerio Público (el análisis paradójicamente se realiza en concordancia a los principios de la constitución 1979 que por los sucesos del cinco de abril no tardaría en ser sustituido a futuro). Véase BRAMONT ARIAS, Luis A. "Los principios rectores del nuevo Código Penal». Derecho, n. 46. Lima, diciembre 1992, pp. 13-31.

33 San MaRtín CASTRO, César "Tráfico ilícito de drogas: represión, Poder Judicial y Policía». Derecho, n. ${ }^{\circ}$ 46. Lima, diciembre 1992, pp. 149-180.

34 Según el razonamiento del autor, esta teoría tiene validez al constatarse penas de expatriación, expulsión de extranjeros $y$, sobre todo, unas cuestionadas exclusiones de beneficios de implicados en terrorismo y narcotráfico entre otras sin afán "resocializador». Véase Prado Saldarriaga, Victor. "La función de la pena en el Código Penal de 1991". Derecho, n. ${ }^{\circ} 46$. Lima, diciembre 1992, pp. 101-112.

35 VILLAVICENCIO TERREROS, Felipe "Ley penal y hecho punible en el Código Penal peruano de 1991". Derecho, n. ${ }^{\circ} 46$. Lima, diciembre 1992, pp. 33-61. 
Facultad de Derecho. Asimismo, se había decido incluir, en el sistema de cursos por dictarse, la materia Derecho de la Competencia, lo que fue un motivo para dedicar este número a temas de esa especialidad, al mismo tiempo que los alumnos obtendrían un material de consulta de primer nivel.

Quedara para el trayecto editorial de Derecho un apreciable aporte enviado por la Oficina Internacional de la Organización Mundial de la Propiedad Intelectual (OMPI) en su calidad de órgano especializado de la Naciones Unidas, ${ }^{36}$ además de la participación del entonces miembro del Consejo Editorial Baldo Kresalja, quien en única participación hasta el momento colaboró con un análisis sobre la competencia desleal y los criterios adoptados en la regulación peruana al respecto. ${ }^{37}$

\section{Número 48}

Para la siguiente edición se cumplían cinco décadas de creación de Derecho. Pero la conmemoración tenía un cariz muy especial como queda grabado en el apartado «De los Editores»:

En la historia de las instituciones existen años cabalísticos en los que se produce la coincidencia de la celebración de aniversarios especiales; en este año, por ejemplo: el primer año de la vigencia de la Constitución de 1993, los diez años del Código Civil, los cincuenta años de nuestra revista Derecho y los setenta y cinco años que nuestra Facultad de Derecho está celebrando. ${ }^{38}$

En efecto, la salida de Derecho 48, perteneciente a diciembre de 1994, reflejaba cinco décadas de producción académica desde su fundación en 1944, acompañada, además, con el marco de la celebración institucional

36 Este trabajo presenta las funciones de este organismo de Naciones Unidas como promotor de la transferencia de tecnología, relacionada con la propiedad industrial, a países en vías de desarrollo, como es el caso del Perú. Además, se analizan tratados importantes en materia de Derecho Competencial Internacional como son el Convenio de París de 1883 que nació para proteger la propiedad intelectual, el tratado de Cooperación en Materia de Patentes de 1970 y el Arreglo de Madrid, entre los más importantes. Véase OFICINA INTERNACIONAL DE LA OMPI. "Principales Tratados en materia de propiedad industrial administrados por la OMPI». Derecho, n. ${ }^{\circ}$ 47. Lima, diciembre 1993, pp. 87 139.

37 KRESALIA R., Baldo. «Comentarios al decreto ley 26122 sobre represión de la competencia desleal». Derecho, n. ${ }^{\circ}$ 47. Lima, diciembre 1993 pp. 13-86. Este estudio se encuentra relacionado con del análisis que se hiciera en ocasión de la reciente promulgación del decreto ley 26122, con un afán crítico a la casi histórica usanza de adaptar sistemas legales que nos son ajenos en tanto la realidad a la cual se trata de adecuar. Mucho más aún si el razonamiento seguido por le decreto tiene raíces en la legislación española sobre competencia desleal, que pertenece a un sistema europeo de desarrollo económico y social, muy dispar al peruano.

* "De los editores". Derecho, n. ${ }^{\circ} 48$. Lima, diciembre 1994, p. 7. 
de la Facultad misma. En esta fecha se pudo apreciar un apartado denominado "Sección Aniversario», en el que se encuentran el discurso del entonces decano de Facultad, doctor Lorenzo Zolezzi, quien resalta la gran responsabilidad que se encuentra en los artículos de Derecho fruto de la perspectiva propia de profesionales formados en la Pontificia Universidad Católica del Perú. ${ }^{39}$ Se debe añadir, al igual que el discurso que pronunció también el ex decano, doctor Jorge Avendaño, el recuerdo, como parte de la historia de la Facultad, a la memoria de los fundadores, del ex decano Hugo Piaggio y del secretario de Facultad Xavier Kiefer-Marchand. ${ }^{40}$

La sección central de esta entrega se conformó por artículos referidos al Derecho Procesal Civil. Un muy puntual análisis del Código Procesal peruano sobre sus artículos 426 y 427 referidos a la postulación del proceso y sus evidentes cambios con su inmediato referente (Código de Procedimientos Civiles) es el tema que aborda Carlos Parodi Remón. ${ }^{41} \mathrm{La}$ "legitimidad para obrar", su diferencia para con el derecho material y el «interés para obrarn, además de sus características en la legislación peruana -como la de ser el presupuesto de la pretensión y la contradicción-, son revisadas por Fausto Viale Salazar ${ }^{42}$ y se complementan con el estudio procesal sobre la casación civil, artículo presentado por el ex presidente de la Corte Suprema de Justicia y profesor de la Facultad de Derecho, Francisco Velasco Gallo. ${ }^{43}$

Las crónicas anuncian como nota resaltante que, como parte de las actividades, se rindió el homenaje a los 13 ex decanos que pasaron por la Facultad de Derecho desde Carlos Arenas y Loayza (1919-1934) hasta Jorge Avendaño Valdez (1987-1993). La edición de Derecho 48 fue la última bajo la dirección de César Landa. Su impresión data de junio de 1995, y también fue la penúltima que realizaba la Editorial e Imprenta DESA. ${ }^{44}$

39 «Discurso del decano Lorenzo Zolezzi por los setenta y cinco años de la Facultad». Sección Aniversario. Derecho, n. ${ }^{\circ} 48$. Lima, diciembre 1994, pp. 295-301.

* "Discurso del ex decano Jorge Avendaño por los setenta y cinco años de la Facultad", Derecho, n. ${ }^{\circ} 48$. Lima, diciembre de 1994, pp. 299-302.

41 Parodi Remón, Carlos. "Comentarios al Código Procesal Civil. La postulación del proceso». Derecho, n. ${ }^{\circ}$ 48. Lima, diciembre 1994, pp. 13-28.

42 VIALE SAlAZAR, Fausto. "Legitimidad para Obrar". Derecho, n. ${ }^{\circ} 48$. Lima, diciembre 1994. pp. 29-49.

43 Velasco Gallo, Francisco, "La casación civil». Derecho, n. ${ }^{\circ}$ 48, pp. 51-56.

4 Los diversos cambios de presentación de los que fue materia la publicación a partir de la edición siguiente (Derecho PUC, n. ${ }^{\circ} 49$ ) se iniciaron bajo el trabajo de imprenta y diagramación de "Copias Gráficas $S$. A" quien por única vez se encargó de tal labor. La edición número 50 le fue encargada por última vez a Editorial e Imprenta DESA, porque, a partir del número siguiente hasta la última entrega en la actualidad, la labor le fue encomendada a Tarea Asociación Gráfica Educativa. 


\section{Número 49}

Ya con la nueva denominación compuesta y su nueva presentación, Derecho PUC 49 no privilegió algún tema sobre otros. Aprovechó esta edición para poder mostrar trabajos sobre las variadas áreas que abarca el Derecho y exponer a los diversos especialistas con los que la Facultad cuenta para cada una de estas.

De esta forma, se contó con el aporte de Domingo García Belaunde, con una obra que fue presentara en Derecho 35 (diciembre 1985) solo que ahora en una versión complementada y corregida: "Cómo estudiar Derecho Constitucional (una década más tarde)». ${ }^{45} \mathrm{~A}$ esta siguió el artículo de quien fuera ministro de Estado, además de diputado nacional, Carlos Blancas Bustamante, quien, desde la óptica laboral, señaló anotaciones sobre la ley 26513 que modificaba la Ley de Fomento de Empleo al establecer nuevas reglas para los despidos, reformas consentidas por la amplia redacción de la Constitución Peruana de 1993 al respecto. ${ }^{46}$

Lorenzo Zolezzi Ibárcena, quien fue director del Departamento de Derecho y Decano de la Facultad se ocupó del Consejo Nacional de la Magistratura (entonces una institución de lustre, hoy venida a menos). Zolezzi lleva a cabo una revisión de los regímenes anteriores a su establecimiento en la Carta de 1993 —resaltando el provisional "Jurado de Honor» creación del Congreso Constituyente-. Emprende estudios diversos, comentarios sobre la crisis de la judicatura, y su estrecha relación con la carencia de candidaturas a las plazas judiciales y fiscales de aspirantes. ${ }^{47}$

Se contó también para esta edición con el discurso que pronunció Fernando de Trazegnies Granda con ocasión de su incorporación como miembro de la Academia Peruana de la Lengua, en el que proclama «un humilde pero apasionado amor por la lengua y la literatura». El maestro universitario procura redefinir la conexión entre la Literatura y el Derecho, disciplina hoy incorporada en el plan de estudios de numerosas universidades de occidente: «[...] viéndolo desde afuera, la Literatura nos entrega una visión viva del Derecho; frente a una consideración técnica y fría del Derecho,

45 GarCía Belaunde, Domingo. "Cómo estudiar Derecho Constitucional (una década más tarde)". Derecho PUC, n. ${ }^{\circ}$ 49. Lima, diciembre 1995. pp. 5-22. La versión primaria fue: García Belaunde, Domingo "Cómo estudiar Derecho Constitucional)" Derecho, n. ${ }^{\circ}$ 39. Lima, diciembre 1985, pp. 177-189.

* Blancas Bustamante, Carlos. "El despido en la reforma de la ley de fomento del empleo". Derecho, n. ${ }^{\circ} 49$, pp. 187-209.

47 ZOLEZZ! IBARCENA, Lorenzo. «El Consejo Nacional de la Magistratura». Derecho, n. 49 , pp. 123-137. 
visto desde el propio Derecho, la Literatura lo captura para devolvérnoslo con más carne, con más humanidad». ${ }^{48}$

En cuanto a criterio de publicación respecta, cabe señalar que, por primera vez, tal vez como una forma de universalismo, Derecho PUC 49 optó por presentar el primer trabajo de un autor extranjero en su lengua nativa, el inglés. Este fue el caso del profesor británico y director del Instituto de Investigación de la Universidad de Warwick (Reino Unido) Mike McConville. ${ }^{49}$ Esta operación, como veremos, será reiterada sobre todo en la edición subsiguiente.

\section{Número 50}

En la conformación de la edición Derecho PUC 50 se optó por proseguir con el criterio de no abordar un tema de Derecho específico a manera de sección central. Sin embargo, la tendencia estuvo enfocada hacia investigaciones de contexto constitucional y civil predominantemente. De esta manera, esta edición fue la correspondiente a diciembre de 1996, con una salida de imprenta que data de febrero de 1998. Este último aspecto generaba el reto al que se sometía el Consejo Editorial con el fin de no solo alcanzar que la entrega anual se regularice sino de no caer en los riesgos de incrementar la diferencia ya existente entre la salida concreta de la publicación impresa y el año al que estaba dedicada el ejemplar en cuestión.

Derecho PUC 50 contó con los valiosos apoyos de los profesores de la facultad como fue el caso de Ricardo Herrera Vásquez, que analizó el contexto creado por la Carta de 1993 respecto a la peculiaridad de los decretos de urgencia como medidas extraordinarias dotadas de fuerza de ley. ${ }^{50} \mathrm{El}$ editor general Aníbal Quiroga también realizó un aporte con un análisis de los sistemas americano y europeo de control difuso y concentrado, respectivamente, y su asimilación dentro del constitucionalismo peruano; este au-

46 De Trazegnies Granda, Fernando. "El derecho como tema literario. Discurso de incorporación a la Academia Peruana de la Lengua». Derecho PUC, n. ${ }^{\circ} 49$, p. 366.

49 MCCONVILLE, Mike "The Origins of the Institutionalized Guilty Plea in the United States: Some Lessons from he History». Derecho PUC, n. ${ }^{\circ} 49$, pp. 87-97. En adelante, en las siguientes ediciones, sucesivos trabajos foráneos en algunos casos también irán acompañados de traducciones al español realizadas bajo la revisión del Consejo Editorial.

so HeRreRA VÁsquez, Ricardo. "Las medidas extraordinarias y los derechos de urgencia en el modelo constitucional peruano". Derecho PUC, n. ${ }^{\circ}$ 50. Lima, diciembre 1996, pp. 235-255. El profesor Ricardo Herrera, especialista en el área de Derecho Laboral en la Facultad de Derecho, con motivo de sus estudios en la Maestría de Derecho Constitucional en la misma universidad, abarca esta área de investigación. 
tor relaciona su investigación con la labor y características del Tribunal Constitucional. ${ }^{51}$

Destaca el estudio que acomete Carlos Fernández Sessarego sobre el tema del daño al proyecto de vida - que fuera su polémico aporte al Código Civil de 1984-, la sintomalogía y sus consecuencias, la protección jurídica en distintos ordenamientos y la posición que adopta el ordenamiento nacional. ${ }^{52}$ En esa misma entrega, se observa un instructivo artículo sobre la figura del fideicomiso a cargo de Francisco Avendaño Arana. ${ }^{53}$

\section{Número 51}

Derecho PUC 51 destaca como entrega por dos detalles fundamentales: se retomó la práctica de proponer un tema específico para su análisis en una sección central —que en este caso fue la garantía constitucional del hábeas data - $y$, en segundo lugar, destaca en esta edición el considerable aporte de material extranjero, una muestra de la difusión y la importancia de $D e-$ recho PUC más allá del ámbito nacional. En palabras del editor general, esta edición simbólicamente representaba un fortalecimiento en el intercambio de información y conocimiento para con otras facultades de derecho iberoamericanas, europeas y norteamericanas que encarnaban, al mismo tiempo, más canales receptores para que el fruto de la investigación local sea conocida en otros ámbitos, lo que sin duda fortalecía más al proyecto. La representación de diversas facultades de derecho iberoamericanas se reflejaban en los importantes autores que colaboraron con las distintas realidades y concepciones de la figura del hábeas data: de Brasil, la obra del catedrático y decano de la Universidad de São Paulo, Dalmo de Abreu Dallari; ${ }^{54}$ de Colombia, con el trabajo del presidente de la Corte Constitucional Colombiana, Eduardo Cifuentes Muñóz; ${ }^{5}$ de Bolivia con la obra de quien fuera ministro, senador y alcalde de La Paz, además de ex profesor y decano de la Universidad Mayor de San Andrés, Benjamín Miguel Harb; ${ }^{56}$ la experien-

51 QUIROGA LEÓN, Aníbal "Control difuso y control concentrado en el derecho procesal constitucional peruano" Derecho PUC, n. ${ }^{\circ}$ 50. Lima, diciembre 1996, pp. 207-233.

¿2 FeRnánDez SeSSAREGo, Carlos. "Daño al proyecto de vida». Derecho PUC, n. ${ }^{\circ}$ 50, pp. 4997.

53 Avendaño A. Francisco. "El fideicomiso». Derecho PUC, n. ${ }^{\circ}$ 50, pp. 343-365. Este artículo, señala el autor a pie de página, representa una actualización de una versión preparada en 1994 para el Grupo Peruano de la Asociación Henry Capitant.

54 De ABreu DallarI, Dalmo. "O hábeas data no sistema jurídico brasileiro". Derecho PUC, n. ${ }^{\circ}$ 51. Lima, diciembre 1997, pp. 95-113. Versión en portugués.

55 CIFuentes MuÑOZ, Eduardo. «El hábeas data en Colombia». Derecho PUC, n. ${ }^{\circ}$ 51, pp. 115-144.

` HARB, Benjamín Miguel. «El hábeas data en Bolivia». Derecho PUC, n. ${ }^{\circ}$ 51, pp. 145-148. 
cia argentina la relata el catedrático de las universidades Nacional de Buenos Aires y Católica de Rosario, Néstor Pedro Sagués, ${ }^{57}$ Oscar Raúl Puccinelli ${ }^{58}$ de la Universidad Católica Argentina, y Roberto Berizonce ${ }^{59}$ de la Universidad Nacional de la Plata. La práctica sureña aparece descrita y comparada con otras latitudes en la obra de Francisco Zúñiga Urbina ${ }^{60}$ de la Universidad de Chile y, en el ámbito nacional, por el doctor Christian Suárez Crothers ${ }^{61}$ para finalizar con dos representantes españoles, Francisco Fernández Segado (Universidad de Compostela y Santiago de Compostela) ${ }^{62}$ y Miguel Revenga Sánchez, de la Universidad de Cádiz. ${ }^{63}$

\section{Número 52}

La promulgación, el 29 de octubre de 1997, y posterior entrada en vigencia de la ley 26872, - Ley de Conciliación - generarían sin duda, el estudio de esta figura procesal por su relevancia al disponerse como etapa previa al litigio judicial con el fin de buscar alternativas de solución que evadan el tradicional y dilatado camino de la justicia ordinaria. La edición Derecho PUC 52, correspondiente a diciembre 1998-abril 1999, estuvo consagrada a publicar predominantemente análisis y estudios respecto a este tema, debido a su reciente aparición.

Los conceptos básicos fueron didácticamente expuestos por el profesor César Guzmán Barrón, ${ }^{64}$ quien en ese momento formaba parte del Consejo Editorial. Asimismo, la teoría de los conflictos, acompañada de un análisis de sus elementos y su relación con la actitud que deben de tomar los abogados frente a estas son vistas por el profesor Fabián Pérez Núnez, ${ }^{65}$ a

$\varpi$ SACUÉs, Néstor Pedro. «El Hábeas Data argentino (Orden Nacional)». Derecho PUC, n. ${ }^{\circ}$ 51, pp. 177-191.

\$) PuCCINELLI, Oscar Raúl. «El hábeas data en las provincias argentinas y en la ciudad autónoma de Buenos Aires". Derecho PUC, n. ${ }^{\circ}$ 51, pp. 149-176.

9 BERIzONCE, Roberto O. "Las grandes líneas tendenciales del proceso civil a fines del segundo milenio». Derecho PUC, n. ${ }^{\circ}$ 51, pp. 311-322.

6o ZúNIIGA URBINA, Francisco. "Derecho a la intimidad y Hábeas Data (del recurso de protección al Hábeas Data)». Derecho PUC, n. ${ }^{51}$, pp. 193-322.

6 SUÁREZ CROTHERS, Christian. «Informática, vida privada y los proyectos chilenos sobre protección de datos». Derecho PUC, n. ${ }^{\circ}$ 51, pp. 223-289.

62 FeRnÁNDEZ SEGADO, Francisco. «El régimen jurídico del tratamiento automatizado de datos de carácter personal en España». Derecho PUC, n. ${ }^{\circ}$ 51, pp. 7-48.

63 RevenGa SÁNCHEZ, Miguel. "El acceso a la información reservada por motivos de seguridad nacional en los Estados Unidos Un balance de la aplicación de la Freedom of Information Act». Derecho PUC, n. 51, pp. 61-94.

64 GUZMÁN BARRÓN, César. «La conciliación: principales antecedentes y características». Derecho PUC, n. ${ }^{\circ}$ 52. Lima, diciembre 1998-abril 1999, pp. 67-74.

65 PéREZ NúÑez, Fabián. "La idea del conflicto y el rol del abogado frente a él». Derecho PUC, n. ${ }^{\circ}$ 52, pp. 189-196. 
quien asiste la experiencia de pertenecer al Centro de Conciliación de la Pontificia Universidad Católica del Perú.

Como bien señalamos, una revisión de la ley en sus principios fue el foco del trabajo del profesor Javier la Rosa Calle, ${ }^{66}$ quien ejerce la cátedra de Negociación y Conciliación de la Facultad de Derecho, además de tener el cargo de Director de Proyectos del Instituto Peruano de Resolución de Conflictos, Negociación y Mediación (IPRECON). Iván Ormachea Choque, presidente del mencionado instituto, analiza la conveniencia de circunscribir la "violencia familiar" como materia conciliable y describe las inseguridades que ofrecería sin las medidas de protección a los afectados. Al mismo tiempo, esta edición nos daba cuenta, en la Crónica del Claustro, de que el proceso de elección del nuevo Decano de la Facultad de Derecho había favorecido al tributarista arequipeño Armando Zolezzi Moller, elegido por la junta de profesores. Se indica también que el presidente de Venezuela, el jurista Rafael Caldera, recibía la distinción como Doctor Honoris Causa. ${ }^{67}$

\section{Número 53}

Uno de los volúmenes más extensos de la historia de esta publicación (edición de 1072 páginas), Derecho PUC 53, perteneciente a diciembre del año 2000, se terminó de imprimir en enero del 2002. La presentación de esta edición afirma, en palabras de su editor general, una «etapa de transición política, y de análisis de nuestras instituciones constitucionales». El panorama político daba cuenta de la crisis que para el año 2000 - tras un brevísimo periodo transcurrido en el gobierno- hace dimitir del cargo al entonces presidente Fujimori en medio de las críticas producidas por un régimen carente de legitimidad. Justamente, este panorama es uno de los temas tocados por el profesor César Delgado-Guembes ${ }^{68}$ dentro de una de las expresiones más comunes del pasado régimen: el "transfugismo", como fenómeno parlamentario cuya erradicación se propugna como parte de una etapa de reinstitucionalización democrática. El mismo escenario es examinado por el profesor César Ochoa Cardich, quien plantea alternativas de reforma de orden constitucional para replantear el régimen político calificando en

66 LA ROSA CALLE, Javier. "Los principios de la conciliación y la ley $N^{\circ} 26872 »$. Derecho PUC, n. ${ }^{\circ}$ 52, pp. 107-117.

67 "Crónica del Claustro». Derecho PUC, n. ${ }^{\circ}$ 52, pp. 1032-1033.

6s Delgado-GuemBes, César. "Transfuguismo y crisis postelectoral en el proceso reinstitucionalización democrática». Derecho PUC, n. ${ }^{\circ}$ 53. Lima, diciembre 2000, pp. 89132. 
sus propios términos como «monarquía presidencial" la versión planteada por el Constituyente de $1993 .{ }^{69}$

Se incluye, además, un aporte que encajaba perfectamente dentro de la actualidad en ese momento, vivida pero desde un plano reflexivo: "Caracterización y problemática del Presidencialismo Iberoamericano ${ }^{70}$ es un trabajo perteneciente a la licenciada en Derecho de la Facultad de Jerez de la Frontera-Universidad de Cádiz, María de la Paz Sánchez. Ella, entre otros aspectos, analiza los poderes y restricciones inherentes a los presidentes en Latinoamérica y complementa su análisis con una remisión a las Constituciones de este lado del mundo; confronta y examina las los poderes legislativos, administrativos, el manejo de la política exterior, etcétera, además de las relaciones entre el poder ejecutivo y el legislativo, y las responsabilidades políticas de los ministros.

En otras áreas, se encuentran trabajos como «Causales de extinción de la acción penal y de la ejecución de la pena ${ }^{71}$ de Víctor Prado Saldarriaga, quien ostenta el grado de doctor en Derecho por la Universidad de Valencia de España, además de ser profesor en la Facultad de Derecho de la Universidad Católica y Universidad Nacional Mayor de San Marcos. También se registra el trabajo histórico-constitucional del profesor Juan Carlos Morón Urbina, quien colaboró con el tema «Bolívar y su propuesta constitucional de $1826{ }^{72}$ Se publica también el aporte en materia de Derecho Civil del doctor Carlos Fernández Sessarego, «Repensando el Código Civil peruano de 1984 en el umbral del nuevo milenion, ${ }^{73}$ entre otras no menos importantes publicaciones que se reunieron para esta entrega.

La reconstitución del orden democrático nacional traía consigo una nueva etapa de renacimiento institucional y social para el Perú. La Comisión de la Verdad y Reconciliación se erigió así como un proyecto que, al intentar aclarar las graves violaciones de derechos humanos ocurridas entre 1980 y 2000 , buscó fortalecer el régimen democrático recuperado, pues era una tarea que no correspondía solamente a un sector sino a la sociedad civil en general.

OCHOA CARDICH, César. «Propuesta de reforma constitucional del régimen político peruano para la reinstitucionalización democrátican: Derecho PUC, n. ${ }^{\circ}$ 53, pp. 313-344.

to Sánchez Manzano, María de la Paz. "Caracterización y problemática del presidencialismo iberoamericano». Derecho PUC, n. ${ }^{\circ}$ 53, pp. 267-312.

$\pi$ PRAdo SaldarRiaca, Víctor. "Causales de extinción de la acción penal y de la ejecución de la pena". Derecho PUC, n. ${ }^{\circ}$ 53, pp. 905-947.

7 MORÓN URBINA, Juan Carlos. «Bolívar y su propuesta constitucional de 1826». Derecho PUC, n. ${ }^{\circ}$ 53, pp. 133-172.

3 Fernández Sessareco, Carlos. «Repensando el Código Civil peruano de 1984 en el umbral de un nuevo milenio» Derecho PUC, n. ${ }^{\circ}$ 53, pp. 373-422. 


\section{Número 54}

En fechas en las cuales acontecían estos hechos, se fue elaborando Derecho PUC 54, cuya edición correspondió a diciembre del 2001. Entre los aportes que pudo reunir, tuvo especial cabida el discurso de instalación de la Comisión de la Verdad que pronunciara su director, el doctor Salomón Lerner Febres, quien era, al mismo tiempo, el Rector de la Pontificia Universidad Católica del Perú. Él representaba a la casa de estudios en un acontecimiento como este, tan gravitante en el suceder nacional. En ese discurso, se manifiesta en qué consistiría la labor de la comisión cuya dirección se le había conferido:

Comprendemos que no somos jueces ni fiscales y que nuestra tarea es retirar los velos que hasta hoy encubren o disfrazan episodios vergonzosos de nuestro pasado que no deben repetirse jamás. Si algo quisiéramos que se diga de nosotros al final de la tarea, es que hemos contribuido a fortalecer el auténtico espíritu cívico de nuestra patria, que no es sino la capacidad de vivir en un clima de respeto mutuo y de apego a la ley. ${ }^{74}$

El consejo editorial decidió asumir como tema central para esta edición los derechos fundamentales y su protección constitucional. Así, se contó con gran colaboración extranjera, aspecto que se constata con el aporte del profesor venezolano Carlos Ayala Corao (quien fuera, además, presidente de la Comisión Interamericana de Derechos Humanos)..$^{75}$

En mayo de 2001, la Facultad de Derecho otorgó, en mérito a sus notables pergaminos, la distinción de Doctor Honoris Causa a Héctor Fix Zamudio, jurista mexicano que ocupara la presidencia de la Corte Interamericana de Derechos Humanos y que también colaboró con esta edición con su artículo «Reflexiones sobre la función constitucional de la oposición política en el ordenamiento mexicano». ${ }^{76}$

74 LeRner Febres, Salomón. «Discurso con ocasión de la Instalación de la Comisión de la Verdad y Anexos". Derecho PUC, n. ${ }^{\circ}$ 54. Lima, diciembre 2000, pp. 493-502.

7 AyAla CORAO, Carlos. "Amenazas judiciales a la libertad de expresión en Venezuela". Derecho PUC, n. ${ }^{\circ}$ 53, pp.7-61. Se analiza el caso particular de la sentencia 1013 de la Sala Constitucional de Venezuela que declaró improcedente un amparo contra el presidente Hugo Chávez al no admitirse al ciudadano Elías Santana el derecho constitucional de réplica hacia comentarios ejercidos en el programa estatal Aló Presidente.

* FIX-ZAMUdio, Héctor. "Reflexiones sobre la función constitucional de la oposición política en el ordenamiento mexicano". Derecho PUC, n. ${ }^{\circ}$ 54, pp. 141-418. En este artículo se analizan la participación activa y las prerrogativas de las agrupaciones políticas mexicanas de oposición que, a partir de las reformas constitucionales de 1994 y 1996, refirmaron la pluralización política que destruyó años de tradicional mayoría del Partido Revolucionario Institucional (PRI). 


\section{Número 55}

El apartado editorial de la edición Derecho PUC 55, de diciembre de 2002, iniciaba con una presentación que transmite las adversidades contra las cuales se debía de batallar para lograr la publicación. El editor Aníbal Quiroga manifestaba los reveses existentes que no lograron detener la labor de conformación de edición: "Dentro de un contexto político y social a veces angustioso, y dentro de una fragilidad económica, en un medio a veces hostil y desalentador que no facilita ni el trabajo adecuado, ni la creatividad, ni la lucidez [...]». Sin embargo, con el apoyo del Fondo Editorial de la Universidad Católica, que en todas las ediciones anteriores fue motivo de gratitudes por parte del Consejo Editorial, y también gracias a las autoridades administrativas de la Facultad de Derecho y al Rector de la Universidad, este proyecto se sobrepuso a sus problemas y pudo presentar esta edición, cuya salida de imprenta se realizó en julio de 2003.

En esta edición se puede ver el aporte de textos en lenguas extranjeras junto con sus respectivas traducciones al español. De esta manera y con un esfuerzo añadido (en la faceta de traducción) que realizaba el Consejo Editorial, quedarán como obras importantes los aportes del profesor de Derecho Público y Constitucional (Universitá Degli Studi di Foggia y Universitá di Roma-Italia) Alfonso Celotto y de la doctoranda por la Universidad Paris XX Nanterre (Francia) Ana Djoric, quienes presentaron, respectivamente, los trabajos «Parlamento y Poder Legislativo del Gobierno en Italia: el abuso del decreto de urgencia" ${ }^{77}$ y "Las instituciones de la Unión Europea: el estatus, la eficacia y equilibrio institucional de cara a su expansión". ${ }^{78}$

$\mathrm{El}$ aporte nacional en la investigación "Génesis, desarrollo, status y futuro proyecto de armonización de las Normas Trasnacionales del Proceso Civil $\gg^{79}$ es fruto de la investigación de Aníbal Quiroga como becario en el UNIDROIT de Roma, trabajo que fuera inicialmente concebido en lengua francesa y que es presentada además en español e inglés.

7 Celotro, Alfonso. "Parlamento e poteri legislativi del Governo in Italia: I'a abuso del decreto d'urgenza (decreto-legge)». Derecho PUC, n. ${ }^{\circ} 55$. Lima, diciembre 2002, pp. 5595.

> DJORIC, Ana. "Les Institutions de l'Union Europeenne: Le Status, les competences et l'equilibre institutionnel en face de l'elargissement». Derecho PUC, n. ${ }^{\circ}$ 55, pp. 113-154.

79 Quiroga León, Aníbal. "Genèse, Développement, "Status" et Futur du Projet D'harmonisation des Normes Transnationales de la Procédure Civile». Derecho PUC, n. ${ }^{\circ} 55$, pp. 245-322. 


\section{Número 56}

Para julio de 2004 se procuraba salida de la última entrega, hasta hoy, de la revista. La voluminosa edición con la que se cerraba la labor encomendada en el ya distante mes de diciembre de 1995 no se dedicó otra vez a un tema central, por lo cual esta edición refleja las constantes características de Derecho PUC en los últimos diez años: se presentaron artículos en diversas áreas del Derecho, acompañados de colaboraciones extranjeras en lenguas originales, una de las cuales posee su traducción al español. ${ }^{80}$

Entre los artículos nacionales destaca "El Convenio arbitral», ${ }^{81}$ obra del ex decano del Colegio de Abogados, Fernando Vidal Ramírez, quien analiza esta particular institución en la Ley General de Arbitraje en sus antecedentes históricos, las oportunidades de celebración del convenio arbitral y la característica de este como posible responsable de la interrupción de la prescripción extintiva. El tema del daño al proyecto de vida, que ya había sido tratado en edición pasada por Carlos Fernández Sessarego, es retomado por el mismo autor bajo una perspectiva más amplia en «El daño al proyecto de vida en la jurisprudencia de la Corte Interamericana de Derechos Humanos" ${ }^{82}$ Destaca el maestro sanmarquino la evaluación que hace de la sentencia de la Corte Interamericana de Derechos Humanos en el caso de María Elena Loayza Tamayo procesada por la justicia militar con el cargo de terrorismo agravado, absuelta por esta pero subsiguientemente vuelta a procesar bajo el mismo cargo pero ahora bajo la jurisdicción común.

Un meritorio análisis de la realidad educativa de las Facultades de Derecho del país se encuentra reflejado en "La enseñanza del Derecho en el Perú: cambios, resistencias y continuidades ${ }^{83}$ que presenta, Gorki Gonzales Mantilla, actual director de esta revista. Imperdible referencia y hasta tópico obligatorio para cualquier estudio sobre el particular.

\$ Este es el caso de los aportes de FERRAND, Frédérique. "La procedure civile internationale et la procedure civile transnationale: l'incidence de l'integración economique regionale". Derecho PUC, n. ${ }^{\circ}$ 56. pp. 297-360; y de la publicación bilingüe de MEZzETI, Luca. "Teoría e Prassi dele Transizioni Constituzionali e del consolidamento Democrático agli inizi del nuovo millenio». Derecho PUC, n. ${ }^{\circ} 56, \mathrm{pp} .29+139$, traducido como «Transiciones constitucionales y consolidación de la democracia en los albores del siglo XXl" por el Consejo Editorial.

3t VIDAL Ramirez, Fernando. "El Convenio arbitral». Derecho PUC, n. ${ }^{\circ} 56$, pp. 569-582.

22 Fernández SeSSAREGo, Carlos. «El daño al «proyecto de vida» en la jurisprudencia de la Corte Interamericana de Derechos Humanos". Derecho PUC, n. ${ }^{\circ}$ 56, pp. 659-700.

a Gonzales Mantilla, Gorki. "La enseñanza del Derecho en el Perú: cambios, resistencias y continuidades". Derecho PUC, n. ${ }^{\circ}$ 56, pp. 880-944. 
Se cierra este modo el último ciclo de Derecho PUC. Sin duda la revista persistirá, tal como perduran las instituciones compactas. Variarán ciertamente los directores, los consejos directivos y sus colaboradores tanto como mudan las cátedras y las generaciones. Cambiarán también las tendencias técnicas, culturales e ideológicas. Los códigos, leyes y Constituciones ya no serán los mismos, en especial en un país caracterizado por la fugacidad normativa y la precariedad general; sin embargo, ya sea el entusiasmo o el escepticismo ante los cambios, se nos antoja siempre retratado desde las páginas de Derecho PUCP. ¿¿Destino imperecedero? Quizá, pero preferiríamos llamarlo estabilidad. 